Part of Journal of Research of the National Bureau of Standards, Volume 29, December 1942

\title{
TEN-YEAR TESTS OF HIGH-EARLY-STRENGTH CEMENT CONCRETES
}

\author{
By Louis Schuman
}

\section{ABSTRACT}

Data on 10-year compressive strengths of concrete cylinders made from 12 commercial high-early-strength cements are given, supplementing the results up to 1 year given in Research Paper 799.1 When cured in moist air, the concretes generally continued to gain strength between 1 and 10 years. For concretes stored in the air of the laboratory, the 10-year strengths are approximately equal to those at 28 days. Comparisons are given between strengths of concretes made in 1910,1930 , and 1941, showing that concretes made with some present-day cements may attain strengths in 1 month exceeding those for the older cements at 10 years.

\section{CONTENTS}

I. Introduction

II. Cements, mixes, and storage conditions

III. Ten-year test results

\section{INTRODUCTION}

Data on the properties of 12 commercial high-early-strength cements, and on mortars and concretes made from them, were published by the National Bureau of Standards in $1935 .^{1}$ The data included compressive strengths of 6 - by 12 -in. cylinders at ages up to 1 year; the present report supplements that of 1935 with data on strengths of concretes at the age of 10 years.

\section{CEMENTS, MIXES, AND STORAGE CONDITIONS}

The chemical compositions and fineness values of the cements are given in table 1. Data on other properties of the twelve cements and on the strengths of the various mortars in which they were incorporated are given in the original report. The concrete mix was $1: 2.28: 4.51$ by weights of dry cement, sand, and gravel, respectively. The sand had a fineness modulus of 2.8. The gravel ranged from $1 / 4$ to $1 \mathrm{in}$. in size. Concretes of three $C / W$ (cement:water) ratios were tested, namely $1.73,1.50$, and 1.33 by weight, corresponding to 6.5 , 7.5 , and 8.5 gal of water per $94-\mathrm{lb}$ bag cement.

${ }^{1} \mathrm{~L}$. Schuman and E. A. Pisapia, Behavior of high-early-strength cement concretes and mortars under various temperature and humidity conditions, J. Research NBS 14, 723 (1935) RP799. 
TABLE 1.-Chemical compositions and fineness values of cements

\begin{tabular}{|c|c|c|c|c|c|c|c|c|c|c|c|c|}
\hline \multicolumn{11}{|c|}{. } & \multicolumn{2}{|c|}{ Fineness } \\
\hline Cement & $\mathrm{CaO}$ & $\mathrm{MgO}$ & $\mathrm{Al}_{2} \mathrm{O}_{3}$ & $\mathrm{Fe}_{2} \mathrm{O}_{3}$ & $\mathrm{SiO}_{2}$ & $\mathrm{SO}_{3}$ & $\mathrm{Cl}$ & $\begin{array}{c}\text { Insol- } \\
\text { uble } \\
\text { in } \\
\mathrm{HCl}\end{array}$ & $\begin{array}{l}\text { Loss } \\
\text { on } \\
\text { igni- } \\
\text { tion }\end{array}$ & $\begin{array}{c}\text { Free } \\
\mathrm{CaO}\end{array}$ & $\begin{array}{c}\text { Re- } \\
\text { tained } \\
\text { on No. } \\
325 \\
\text { sieve }\end{array}$ & $\begin{array}{c}\text { Specific } \\
\text { surface } \\
\text { by } \\
\text { Wagner } \\
\text { turbi- } \\
\text { dimeter }\end{array}$ \\
\hline $\begin{array}{l}1 \\
3 \\
4 \\
5 \\
6 \\
6\end{array}$ & $\begin{array}{l}\% \\
66.2 \\
66.6 \\
65.6 \\
65.7 \\
64.2 \\
62.9 \\
65.7 \\
65.4 \\
66.2 \\
62.3 \\
63.3 \\
60.6 \\
6\end{array}$ & $\begin{array}{l}\% \\
1.1 \\
1.0 \\
2.5 \\
2.5 \\
3.4 \\
2.9 \\
1.5 \\
2.7 \\
2.2 \\
3.4 \\
3.5 \\
4.8 \\
9 \\
\end{array}$ & $\begin{array}{l}\% \\
8.3 \\
6.2 \\
4.7 \\
5.6 \\
7.2 \\
7.0 \\
6.6 \\
6.4 \\
4.4 \\
7.1 \\
6.4 \\
4.8 \\
\end{array}$ & $\begin{array}{l}\% \\
3.1 \\
1.9 \\
3.1 \\
2.3 \\
2.5 \\
2.3 \\
2.7 \\
1.9 \\
2.8 \\
2.8 \\
2.5 \\
2.4 \\
\end{array}$ & $\begin{array}{c}\% \\
17.0 \\
20.6 \\
20.6 \\
19.3 \\
18.5 \\
20.3 \\
19.3 \\
19.3 \\
21.6 \\
18.5 \\
19.1 \\
21.9 \\
20\end{array}$ & $\begin{array}{l}\% \\
2.4 \\
2.5 \\
1.3 \\
2.2 \\
2.4 \\
2.6 \\
2.1 \\
2.3 \\
1.9 \\
2.5 \\
2.6 \\
2.7 \\
\end{array}$ & $\begin{array}{l}\% \\
0.0 \\
.0 \\
.3 \\
.02 \\
.04 \\
.08 \\
.03 \\
.0 \\
.0 \\
.0 \\
.07 \\
.0 \\
\end{array}$ & $\begin{array}{r}\% \\
0.2 \\
.5 \\
.5 \\
.6 \\
.2 \\
2.3 \\
0.4 \\
.2 \\
.1 \\
.5 \\
.4 \\
.7 \\
\end{array}$ & $\begin{array}{l}\% \\
1.7 \\
1.1 \\
2.4 \\
2.1 \\
1.8 \\
1.6 \\
1.8 \\
1.7 \\
1.2 \\
2.7 \\
1.9 \\
2.5 \\
\end{array}$ & $\begin{array}{r}\% \\
0.4 \\
1.6 \\
0.3 \\
2.7 \\
0.6 \\
.1 \\
1.5 \\
4.2 \\
0.4 \\
1.9 \\
1.2 \\
0.9 \\
\\
\end{array}$ & $\begin{array}{l}8.5 \\
8.3 \\
8.8 \\
8.7 \\
5.3 \\
8.4 \\
5.8 \\
9.6 \\
7.5 \\
8.8 \\
5.1 \\
6.6 \\
\end{array}$ & $\begin{array}{r}\mathrm{cm}^{2} / g \\
1,990 \\
1,910 \\
1,770 \\
2,030 \\
1,760 \\
2,050 \\
1,970 \\
2,180 \\
2,120 \\
1,930 \\
2,150 \\
2,490 \\
\end{array}$ \\
\hline
\end{tabular}

a By method of Emley, Trans. Am. Ceram. Soc. 17, 720 (1915).

b Contains a water-repellent material.

The temperatures of the materials at the time of mixing, and the conditions of storage of the test specimens during the first 24 hours after molding, were as follows:

(1) Mixed at $70^{\circ} \mathrm{F}$, stored in air at $70^{\circ} \mathrm{F}$.

(2) Mixed at $90^{\circ} \mathrm{F}$, stored in air at $90^{\circ} \mathrm{F}$.

(3) Mixed at $110^{\circ} \mathrm{F}$, stored in air at $110^{\circ} \mathrm{F}$.

(4) Mixed at $70^{\circ} \mathrm{F}$, stored in a thermally insulated cabinet.

(5) Mixed at $70^{\circ} \mathrm{F}$, stored adiabatically (no heat loss).

No 10-year specimens were made for the adiabatic storage. After the first 24 hours, specimens from each of the other four initial conditions were treated as follows until tested:

(1) Stored in damp room at $70^{\circ} \mathrm{F}$, at relative humidity over 95 percent.

(2) Stored in air of laboratory at $70^{\circ} \mathrm{F}$.

(3) Stored outdoors for 1 year.

(4) Subjected to 300 cycles of freezing and thawing in approximately 15 months.

Specimens for only the first two of these storage conditions remained for 10-year tests.

\section{TEN-YEAR TEST RESULTS}

The compressive strengths of concretes made from each cement are given in table 2, for ages ranging from 1 day up to 10 years. Each value is the average for a set of three cylinders. The maximum deviation from the average was generally less than 10 percent. Agestrength relations for two of the cements (Nos. 7 and 11), representing typical variations, are shown in figures 1 and 2 .

The concretes which were damp-cured showed continued increase in strength, in most cases, between 1 year and 10 years; such gains were of about the same magnitude as between 28 days and 1 year. In a few instances, concretes stored at $70^{\circ} \mathrm{F}$ during the first 24 hours showed slight decreases in strength between 1 year and 10, but in such cases the 
strengths remained practically equal to those of the concretes which were initially stored at higher temperatures or were thermally insulated. Though the 1-day strengths were higher when the concretes were stored
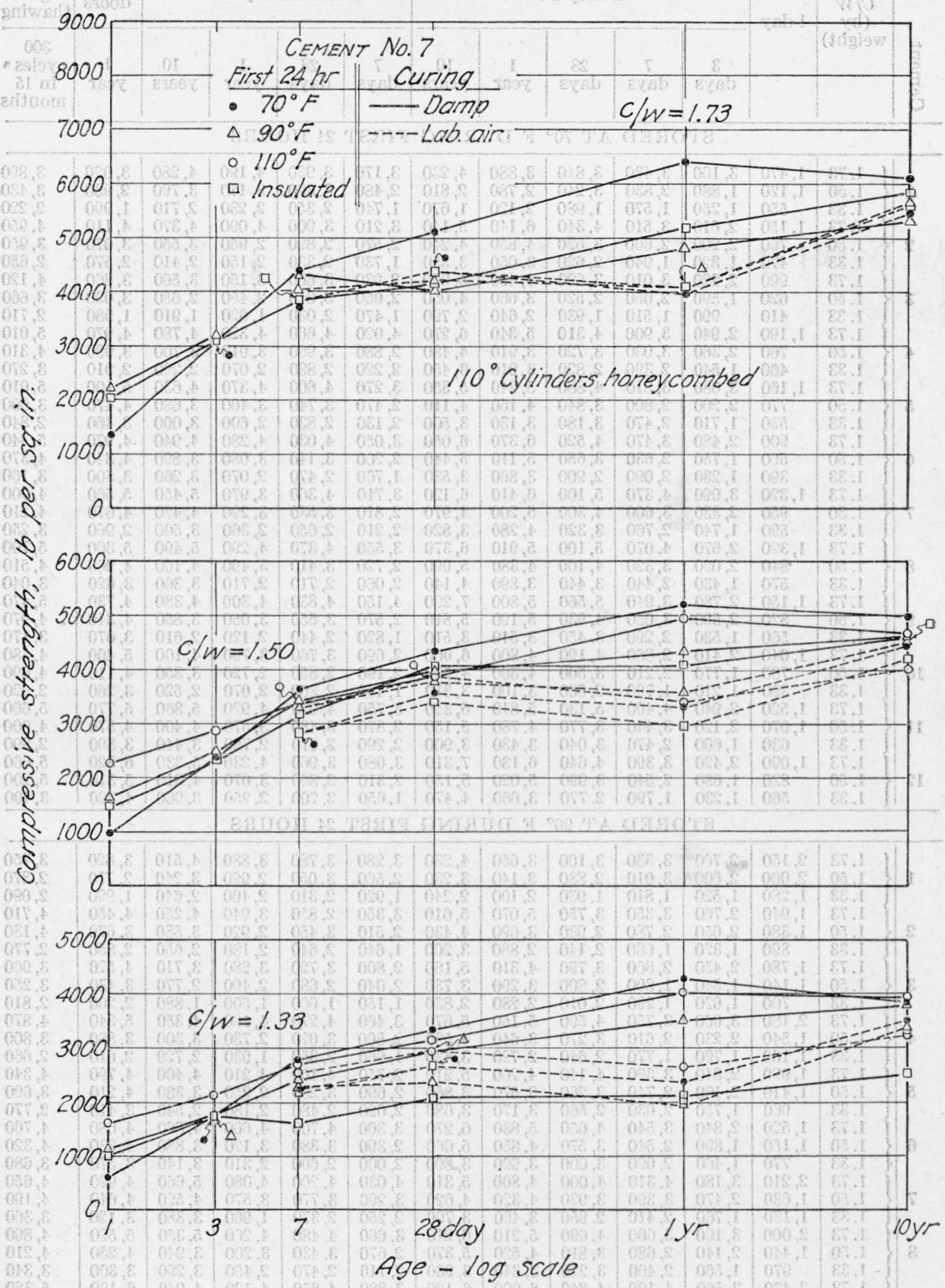

FIGURE 1.-Age-strength relations for cement 7 .

at the higher temperatures during the first 24 hours, the strengths at later ages tend to equalize. There is, however, some tendency for the initially insulated concretes to have somewhat lower strengths after 3 days and up to 10 years than those under the other conditions. 
400 Journal of Research of the National Bureau of Standards

TABLE 2.-Compressive strengths (lb/in.2) of 6-by 12-in. concrete cylinders

\begin{tabular}{|c|c|c|c|c|c|c|c|c|c|c|c|c|c|}
\hline \multirow[b]{3}{*}{$\begin{array}{l}\text { 苞 } \\
\text { घ્ㅇ } \\
0\end{array}$} & \multirow{3}{*}{$\begin{array}{c}\mathrm{ClW} \\
\text { (by } \\
\text { weight) }\end{array}$} & \multirow{3}{*}{1 day } & \multicolumn{11}{|c|}{ Subsequent storage condition } \\
\hline & & & \multicolumn{5}{|c|}{ Damp air } & \multicolumn{4}{|c|}{ Laboratory air } & \multirow{2}{*}{$\begin{array}{c}\begin{array}{c}\text { Out- } \\
\text { doors }\end{array} \\
\begin{array}{c}1 \\
\text { year }\end{array}\end{array}$} & \multirow{2}{*}{\begin{tabular}{|c}
$\begin{array}{c}\text { Freez- } \\
\text { ing and } \\
\text { thawing }\end{array}$ \\
$\begin{array}{c}300 \\
\text { cycles a } \\
\text { in 15 } \\
\text { months }\end{array}$ \\
\end{tabular}} \\
\hline & & & $\begin{array}{c}3 \\
\text { days }\end{array}$ & $\stackrel{7}{\text { days }}$ & $\stackrel{28}{\text { days }}$ & $\begin{array}{c}1 \\
\text { year }\end{array}$ & $\begin{array}{c}10 \\
\text { years }\end{array}$ & $\begin{array}{c}7 \\
\text { days }\end{array}$ & $\stackrel{28}{\text { days }}$ & $\begin{array}{c}1 \\
\text { year }\end{array}$ & $\begin{array}{c}10 \\
\text { years }\end{array}$ & & \\
\hline
\end{tabular}

STORED AT $70^{\circ} \mathrm{F}$ DURING FIRST 24 HOURS

\begin{tabular}{|c|c|c|c|c|c|c|c|c|c|c|c|c|c|}
\hline \multirow{4}{*}{1} & .73 & 470 & 3,100 & 3,430 & 3,840 & 3,880 & 4,230 & 3,170 & 3,930 & 4,190 & 4,280 & 3,020 & 3,890 \\
\hline & 1.50 & 20 & 1,880 & 2,830 & 240 & 780 & 2,810 & 2,480 & 3,390 & 3,140 & 3,790 & 2,930 & 3,430 \\
\hline & 1.33 & 550 & 1,250 & 1,570 & 1,980 & 120 & 1,670 & 1,740 & 2,350 & 2,230 & 2,710 & 1,990 & 2,220 \\
\hline & 1. 73 & 1,150 & 2,610 & 3,510 & 4,340 & 6,140 & 5,140 & 3,210 & 3,900 & 4,090 & 4,370 & 4,110 & 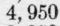 \\
\hline \multirow[t]{3}{*}{2} & 1.50 & 760 & 2,200 & 2,600 & 3,620 & 4,850 & 4,240 & 2,570 & 2,890 & 2,950 & 3,550 & 3,070 & \\
\hline & 1. 33 & 470 & 1,320 & $\mathbf{1}, 940$ & 2,620 & 3,050 & 3,350 & 1,730 & & 2,150 & 0 & & 50 \\
\hline & 1. 73 & 990 & 2,220 & 3,010 & 30 & & & & & 3 , & 0 & & 120 \\
\hline \multirow[t]{3}{*}{3} & 1.50 & 620 & 1,590 & 2,050 & 2,520 & 3,660 & 4,050 & 2,050 & 3,010 & 2,450 & 2,650 & & 3,660 \\
\hline & 1. 33 & 410 & 990 & 1,510 & 1,930 & 2,640 & 2,790 & 1,470 & 2,030 & 1,620 & 1,910 & 1,980 & 10 \\
\hline & 1. 73 & 1,190 & 2,940 & 3,900 & 4,310 & 5,340 & 6,270 & 4,020 & 4,660 & 4,320 & 4,780 & 4 , & \\
\hline \multirow[t]{3}{*}{4} & 1.50 & 760 & 2,360 & 3,030 & 3,720 & 3,910 & 4,480 & 2,880 & 3,960 & 3,010 & 3 , & & \\
\hline & 1. 33 & 460 & 1,540 & 2,390 & 2,820 & 3, & 3,450 & 2 , & 2 , & 2 , & 2 & & \\
\hline & 1. 73 & 1,160 & 3,080 & 3,810 & 30 & 5 , & 5 , & 3 & & 4 , & & & 10 \\
\hline \multirow[t]{3}{*}{5} & 1.50 & 770 & 2,200 & 2,800 & 3,840 & 4,150 & 4,110 & 2 , & 3,740 & 3,400 & & 4 , & 00 \\
\hline & 1.33 & 530 & 1,710 & 2,470 & 3,180 & 3,130 & 3,500 & 2,130 & 2,830 & & 00 & & \\
\hline & 1. 73 & 900 & 2,480 & 3,470 & 4,520 & 6,370 & 6,050 & 3,050 & 4,030 & 80 & 40 & & \\
\hline \multirow[t]{3}{*}{6} & 1.50 & 560 & 1,750 & 2,630 & 3,650 & 5,110 & 5,440 & 2,200 & 3,140 & 3,080 & 3 , & & 0 \\
\hline & 1. 33 & 390 & 1,230 & 2,090 & & 3,860 & 3,530 & 60 & 2,470 & 2 , & 3 , & 3. & \\
\hline & 1. 73 & 1,320 & 3, & 4,3 & 5 , & 6 , & 20 & 3 , & 4 , & 3 , & 5 & 5 , & 4, \\
\hline \multirow[t]{3}{*}{7} & 1.50 & 950 & 2,3 & 3,6 & 4 , & 5 , & 4 & 2 , & 3, & 3,290 & 4 , & 4 , & 0 \\
\hline & 1. 33 & 590 & 1,740 & 2,760 & 3,320 & 4,2 & 3,820 & 10 & 2 , & & & & \\
\hline & 1. 73 & 1,320 & 2,670 & 4,070 & 5,100 & 5,910 & 6,370 & 3,550 & 4,370 & 4,290 & 90 & & \\
\hline \multirow[t]{3}{*}{8} & 1. 50 & 840 & 2,090 & 3,320 & 4,100 & 4,380 & 5,090 & 2,720 & 3.410 & 3,490 & 60 & 4 , & 0 \\
\hline & 1.33 & 570 & 1,430 & 2,440 & 40 & 90 & 4,140 & 2 , & 2 , & 2,710 & 3 & 3 , & 0 \\
\hline & 1. 73 & 1,150 & 2,780 & 3 , & 5 , & 5, & 7,290 & 4,150 & 4,830 & 4,300 & 4, & 4, & 70 \\
\hline \multirow{2}{*}{9} & 1.50 & 820 & 2,590 & 3,050 & 4,930 & 5,190 & 5,810 & 2,570 & 3,550 & 3, & 0 & 10 & 0 \\
\hline & 173 & $\begin{array}{r}560 \\
040\end{array}$ & 1,530 & $\begin{array}{l}2,290 \\
2,860\end{array}$ & $\begin{array}{l}3,450 \\
4,190\end{array}$ & $\begin{array}{l}3,510 \\
4,800\end{array}$ & $\begin{array}{l}3,510 \\
6,060\end{array}$ & $\begin{array}{l}1,820 \\
2,690\end{array}$ & $\begin{array}{l}2,440 \\
3,760\end{array}$ & $\begin{array}{l}2,120 \\
3,790\end{array}$ & $\begin{array}{l}2,610 \\
4,100\end{array}$ & $\begin{array}{l}3,670 \\
5,400\end{array}$ & \\
\hline \multirow[t]{3}{*}{10} & 1.50 & 700 & 1,770 & 2,210 & 3,500 & 4,300 & 4,700 & 2,190 & 2,850 & 2,7 & & & 4,090 \\
\hline & 1.3 & 390 & 1,210 & 1,790 & 2,500 & 3,100 & 3,310 & 1,490 & 2, & 2,0 & 2 , & 3 , & 20 \\
\hline & 1. 73 & 1,520 & 2,940 & 4,400 & 5,130 & 5,810 & 6,340 & 3,750 & 4,920 & 4,920 & 5,860 & 5 , & 5 , \\
\hline \multirow[t]{3}{*}{11} & 1.50 & 1,070 & 2,120 & 3,440 & 3,770 & 4,780 & 5,150 & 2,870 & 3,480 & 3,570 & 4,400 & & 00 \\
\hline & 1. 33 & 630 & 1,600 & 2,470 & 3,040 & 3,430 & 3,900 & 2,290 & 2,670 & 2,790 & 40 & & 90 \\
\hline & 1. 73 & 1,090 & 2,420 & 3,390 & 4,640 & 6,130 & 7,310 & 3,080 & 3,960 & 4,230 & & & 60 \\
\hline \multirow[t]{2}{*}{12} & 1. 50 & 820 & 1,680 & 2,540 & 3,990 & 5,020 & 5,150 & 2,310 & 2,890 & 3,070 & & & 200 \\
\hline & 1. 33 & 560 & 1,230 & 1,790 & 2.770 & 3,660 & 4,470 & 1,650 & 2,200 & 2,250 & 3, & 4,320 & 3,490 \\
\hline
\end{tabular}

STORED AT $90^{\circ} \mathrm{F}$ DURING FIRST 24 HOURS

\begin{tabular}{|c|c|c|c|c|c|c|c|c|c|c|c|c|c|}
\hline & 1. 73 & 2,150 & 2,760 & 3,330 & 3,100 & 3,650 & 4,230 & 3,280 & 3, & 0 & 10 & 3,820 & 3,750 \\
\hline \multirow[t]{3}{*}{1} & 1. 50 & 2,000 & 2,600 & 3,010 & 2,880 & 3,140 & 3,230 & 2,560 & 3,050 & 2,990 & 3,240 & 710 & 370 \\
\hline & 1. 33 & 1,280 & 1,520 & 1,810 & 1,930 & 2,100 & 2,240 & 1,920 & 2,310 & 2,400 & 2,640 & & \\
\hline & 1. 73 & 1,940 & 2,760 & 3,350 & 3,750 & 5,070 & & 3,350 & 2,850 & 3,940 & 4,250 & & 4,710 \\
\hline \multirow[t]{3}{*}{2} & 1. 50 & 1,380 & 2,050 & 2,780 & 2,930 & 3,690 & 4,430 & 2,510 & 3,450 & 2,920 & 3,580 & 3,670 & 4,130 \\
\hline & 1. 33 & 890 & 1,320 & 1,680 & 2,140 & 2,880 & 3,200 & 1,640 & 2,640 & 2,180 & 2,650 & 2,850 & 2,770 \\
\hline & 1. 73 & 1,780 & 2,450 & 2,860 & 3,790 & 4,310 & 5,180 & 2,800 & 3,720 & 3,280 & 3,710 & 4,520 & 3 , \\
\hline \multirow[t]{3}{*}{3} & 1. 50 & 1,140 & 1,540 & $\overrightarrow{1}, 890$ & 2,800 & 3,290 & 3,730 & 2,040 & 2,680 & 2,490 & 2,770 & 3 , & 250 \\
\hline & 1. 33 & 700 & 1,020 & 1,290 & 2,010 & & 2,830 & & & 1 , & 80 & & \\
\hline & 1. 73 & 2,180 & 3,0 & 3 , & 4 , & 5 & 0 & 3,460 & 4 , & 4 , & 4,350 & 5,340 & 4,870 \\
\hline \multirow[t]{3}{*}{4} & 1. 50 & 1,540 & 2,230 & 2,610 & 3,270 & 3,640 & 4,530 & 2,800 & 3,020 & 2,730 & 3,300 & 3,880 & 3,800 \\
\hline & 1. 33 & 1,100 & 1,790 & 1,770 & 2,640 & 2,720 & 3,430 & 1,960 & 2,350 & 1,930 & 2,720 & 2,610 & 2,660 \\
\hline & 1. 73 & 1,990 & 2,840 & 3,390 & 4,140 & 4,260 & 5,210 & 2,750 & 4,160 & 4,210 & 4,400 & 4,790 & 4,340 \\
\hline \multirow[t]{3}{*}{5} & 1. 50 & 1,410 & 2,100 & 2,740 & 3,260 & 3,570 & 3,860 & 2,690 & 3,230 & 2,950 & 3,330 & 4,210 & 990 \\
\hline & 1. 33 & 960 & 1,750 & 2,030 & 2,560 & & & 2,090 & & 30 & 10 & 10 & 2,770 \\
\hline & 1. 73 & 1,520 & 2,840 & 3,5 & 4, & 5 , & 0 & 0 & 4 , & 4 , & 0 & 4, & 4,760 \\
\hline \multirow[t]{3}{*}{6} & 1. 50 & 1,150 & 1,890 & 2,540 & 3,570 & 4,850 & 5,060 & 2,390 & 3, & 3 , & & & \\
\hline & 1. 33 & 770 & 1,460 & 2,090 & 3,0 & & & & 2,600 & 10 & 40 & 570 & 80 \\
\hline & 1. 73 & 2,210 & 3,180 & 4,310 & 4,000 & 4,800 & 5,310 & 4,030 & 4,200 & 80 & 60 & 90 & 50 \\
\hline \multirow[t]{3}{*}{7} & 1. 50 & 1,630 & 2,470 & 3,390 & 3,920 & 4,320 & 4,620 & 3,290 & 70 & 70 & 80 & 40 & 4,190 \\
\hline & & 1,130 & 1,7 & & & & 90 & 50 & 0 & 1, & 3 , & 3 , & 3,360 \\
\hline & 1. 73 & 2,000 & 3,190 & 3,600 & 4, & 5, & 10 & 3 , & 4 , & 4 , & 50 & 5 , & 4,890 \\
\hline \multirow[t]{3}{*}{8} & 1. 50 & 1,440 & 2,140 & 2,680 & 3,810 & 4,520 & 5,370 & 2,670 & 3,430 & 3 , & 40 & 50 & 0 \\
\hline & 1. 33 & 970 & 1,560 & 2,460 & 3,220 & 3,3 & 30 & 110 & 2 , & 50 & 90 & 00 & 40 \\
\hline & 1. 73 & 2,430 & 3,560 & 4,100 & 4,840 & 6, & 20 & & 50 & 20 & 40 & 00 & \\
\hline \multirow[t]{3}{*}{9} & 1. 50 & 1,570 & 2,310 & 2,920 & & & 10 & & & 00 & 10 & 70 & 50 \\
\hline & & 1,060 & 1, & 2,260 & & 0 & 60 & 30 & 2 , & 20 & 2 , & 3, & 3,050 \\
\hline & 1. 73 & 1,590 & 2,160 & 3,050 & 3,670 & 5,170 & 5,680 & 2,890 & 3,650 & 3 , & 4,510 & 4 , & 4,230 \\
\hline \multirow[t]{3}{*}{10} & 1. 50 & 1,240 & 1,800 & 2,140 & 2,990 & 4,040 & 4,560 & 1,970 & 2,710 & 2,810 & 3 , & 00 & 3,830 \\
\hline & 1. 3 & 710 & 1,160 & 1,330 & 2,430 & 2,9 & 3,240 & 1,430 & 1,980 & 2,010 & 30 & 2, & 0 \\
\hline & 1.73 & 2,230 & 3,610 & 3,750 & 5,300 & 5,9 & & 3,930 & & & 10 & & 10 \\
\hline \multirow[t]{3}{*}{11} & 1. 50 & 1,560 & & 3,070 & & & & 2,900 & & & 90 & 80 & 420 \\
\hline & 1. 33 & & 1,440 & 2,220 & 2,6 & 3,4 & 3,570 & 1,950 & 2,730 & 2 , & 3 , & 3 , & 3,060 \\
\hline & 1. 73 & 1,810 & 2,070 & 3,060 & 4,670 & 6,240 & 6,770 & 3,320 & 4,0 & 4,260 & 5,310 & 6 , & 5,260 \\
\hline \multirow[t]{2}{*}{12} & 1. 50 & 1,160 & 1,890 & 2,350 & 3,020 & 4,700 & 5,200 & 2,120 & 2,890 & 3,170 & 4,120 & 4,640 & 4,530 \\
\hline & & 60 & 1,200 & 1,670 & 2,460 & 3,480 & 3,890 & 1,480 & 2,430 & 2,230 & 3,300 & 3,540 & 3,660 \\
\hline
\end{tabular}

a Freezing in air at $5^{\circ}$ to $20^{\circ} \mathrm{F}, 16$ hours; thawing in water at about $70^{\circ} \mathrm{F}, 8$ hours. 
TABLE 2.-Compressive strengths $\left(l b / i n .^{2}\right)$ of 6 -by 12-in. concrete cylinders-Continued

\begin{tabular}{|c|c|c|c|c|c|c|c|c|c|c|c|c|c|}
\hline \multirow[b]{3}{*}{$\begin{array}{l}\text { 范 } \\
\text { 号 } \\
\text { ठ }\end{array}$} & \multirow{3}{*}{$\begin{array}{c}C / W \\
\text { (by } \\
\text { weight) }\end{array}$} & \multirow{3}{*}{1 day } & \multicolumn{11}{|c|}{ Subsequent storage condition } \\
\hline & & & \multicolumn{5}{|c|}{ Damp air } & \multicolumn{4}{|c|}{ Laboratory air } & \multirow{2}{*}{$\begin{array}{c}\begin{array}{c}\text { Out- } \\
\text { doors }\end{array} \\
\begin{array}{c}1 \\
\text { year }\end{array}\end{array}$} & \multirow{2}{*}{$\begin{array}{l}\begin{array}{c}\text { Freez- } \\
\text { ing and } \\
\text { thawing }\end{array} \\
\begin{array}{c}300 \\
\text { cycless } \\
\text { in } 15 \\
\text { months }\end{array}\end{array}$} \\
\hline & & & $\frac{3}{\text { days }}$ & $\stackrel{7}{\text { days }}$ & $\stackrel{28}{\text { days }}$ & $\begin{array}{c}1 \\
\text { year }\end{array}$ & $\begin{array}{c}10 \\
\text { years }\end{array}$ & $\stackrel{7}{\text { days }}$ & $\stackrel{28}{\text { days }}$ & $\begin{array}{c}1 \\
\text { year }\end{array}$ & $\begin{array}{c}10 \\
\text { years }\end{array}$ & & \\
\hline
\end{tabular}

STORED AT $110^{\circ}$ F DURING FIRST 24 HOURS

\begin{tabular}{|c|c|c|c|c|c|c|c|c|c|c|c|c|c|}
\hline & 73 & 620 & 080 & 2,350 & 2,350 & 3,420 & 2,920 & 2,170 & 2,430 & 3,050 & 3,190 & 3,380 & 3,46 \\
\hline \multirow[t]{3}{*}{1} & & 1,780 & 2,190 & 2,680 & 3,100 & 3,550 & 3,220 & 2,310 & 2,910 & 2,960 & 3,210 & 3,540 & 3,490 \\
\hline & 33 & 1,410 & 1,800 & 2,090 & 2,200 & 2,630 & 2,490 & 1,980 & 2,290 & 2,140 & 2,460 & 2,430 & 2,580 \\
\hline & 1. 73 & 2,330 & 2,980 & 2,980 & 3,020 & 4,890 & 5,270 & 3,320 & 3,660 & 3,890 & 4,320 & 4,040 & 450 \\
\hline \multirow[t]{3}{*}{2} & 1. 50 & 1,610 & 2,110 & 2,550 & 2,670 & 4,100 & 4,200 & 2,440 & 3,140 & 2,690 & 3,730 & 3,520 & 770 \\
\hline & 1. 33 & 1,480 & 1,630 & 1,960 & 2,120 & 3,540 & 3,350 & 2,060 & 2,220 & 2,180 & 2,890 & 2,910 & \\
\hline & 1. 73 & 2,470 & 3,010 & 60 & 3,520 & 4, & 5,170 & 3,490 & 4,160 & 4,080 & 4,460 & 4,570 & 810 \\
\hline \multirow[t]{3}{*}{3} & 1. 50 & 1,650 & 2,440 & 2,630 & 2,710 & 3,750 & 4,410 & 2,420 & 2,810 & 2,940 & 3,220 & 3,740 & 10 \\
\hline & 3 & 1,250 & 1,550 & 1,910 & 2,080 & 2,640 & 3,280 & 1,710 & 2,220 & 2,060 & 2,470 & 2,750 & 100 \\
\hline & 1. 73 & 2,360 & 2,490 & 70 & 4,390 & 4,380 & 5,800 & 3,540 & 4,090 & 3, & 4, & 60 & 930 \\
\hline \multirow[t]{2}{*}{4} & 1. 50 & 1,900 & 2,290 & 2 , & 3,420 & 3,980 & $\begin{array}{l}4,520 \\
3\end{array}$ & 2,720 & 3 , & $\begin{array}{l}3 \text {, } \\
2,\end{array}$ & 3 , & $\begin{array}{l}4, \\
3\end{array}$ & 340 \\
\hline & 1. & $\begin{array}{l}1,430 \\
2,300\end{array}$ & $\begin{array}{l}1,810 \\
2,850\end{array}$ & $\begin{array}{l}2,150 \\
2,720\end{array}$ & & 3 , & 3 , & $\begin{array}{l}2,020 \\
2,620\end{array}$ & 2 , & $\begin{array}{l}2,320 \\
3,380\end{array}$ & 3,610 & $\begin{array}{l}3,480 \\
3,500\end{array}$ & $\begin{array}{l}3,340 \\
3,420\end{array}$ \\
\hline \multirow[t]{3}{*}{5} & 1. 50 & 1,660 & 2,130 & 2,430 & 3,010 & 3,520 & 4,280 & 2,560 & 3,010 & 3,120 & 3,360 & 4,020 & 3,570 \\
\hline & 1. 33 & 1,240 & 1,560 & 1,900 & 2,480 & 2,980 & 3,480 & 1,740 & 2,410 & 2,410 & 2,760 & 3,760 & 50 \\
\hline & b 1.73 & 1,640 & 1,530 & 2,240 & 3,070 & 3,460 & 4,100 & 3,170 & 2,910 & 2,840 & 4,110 & 4 , & 50 \\
\hline \multirow[t]{3}{*}{6} & 1. 50 & 1,740 & 2,280 & 2,700 & 3,390 & 4,650 & 5,120 & 2,600 & 3 , & 3,040 & 20 & 90 & 200 \\
\hline & 1. 33 & 1,250 & 1,840 & 2,170 & 3, & 4,250 & 4,600 & 2,120 & 2 , & 2 , & 10 & 30 & 780 \\
\hline & b 1.73 & 1,690 & 2,490 & 2,580 & 2,740 & 3,070 & 3, & 2 , & 4, & 3 , & 90 & 10 & 20 \\
\hline \multirow[t]{2}{*}{7} & 1. 50 & 2,240 & 2,850 & 3,3 & 3, & 4, & 4, & 3,260 & 3,870 & 90 & 00 & 4, & \\
\hline & 1. 33 & 1,600 & 2,120 & 2 & & & 30 & 2,530 & 2 , & & $\begin{array}{l}70 \\
80\end{array}$ & & \\
\hline \multirow{3}{*}{8} & b 1.73 & 2 , & 1,650 & & $\begin{array}{l}3, \\
3,\end{array}$ & $\begin{array}{l}4,050 \\
4,470\end{array}$ & $\begin{array}{l}4,120 \\
4,980\end{array}$ & $\begin{array}{l}3,220 \\
3,050\end{array}$ & $\begin{array}{l}3,300 \\
3,730\end{array}$ & & $\begin{array}{l}5,080 \\
4,600\end{array}$ & $\begin{array}{l}0,080 \\
4,680\end{array}$ & 180 \\
\hline & 1. & $\begin{array}{l}1,970 \\
1,460\end{array}$ & 1,960 & 2,230 & 3,160 & 3,740 & 4,090 & 2,380 & 2,780 & 3,000 & 3,580 & 3,830 & 3,360 \\
\hline & $\begin{array}{l}\text { 1. } 00 \\
1.73\end{array}$ & 2,680 & 3,680 & 4,010 & 4,980 & 5,330 & 6,580 & 3,880 & 4,930 & 4,710 & 5,290 & 6,140 & 5,320 \\
\hline \multirow[t]{3}{*}{9} & 1.50 & 2,290 & 2,840 & 3,500 & 3,650 & 4,580 & 4,890 & 3,500 & 3,910 & 3,750 & 80 & 40 & 550 \\
\hline & 1. 33 & 1,620 & 2,320 & 2,750 & 3,400 & 4,060 & 4,020 & 2,880 & 3 , & & 20 & & 20 \\
\hline & 1. 73 & 1,760 & 2,060 & 2,690 & 3,180 & 3 , & 5,300 & 2,770 & 3 , & 00 & 90 & 90 & 700 \\
\hline \multirow[t]{3}{*}{10} & 1. 50 & 1,650 & 1,770 & 2,170 & 2,560 & 3,330 & 4,200 & 2,140 & 2,730 & 3 , & 3,120 & 4,110 & 770 \\
\hline & 1. 33 & 1,020 & 1,370 & 1,610 & 2,070 & 2,770 & 3,510 & 1,440 & 2,020 & 2 , & 2 , & 3,240 & 330 \\
\hline & 1. 73 & 2,900 & 3,160 & 3,870 & 4,940 & 5,520 & 6,360 & 3,980 & 4,730 & 4, & 6,030 & 5,470 & 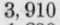 \\
\hline \multirow[t]{3}{*}{11} & 1. 50 & 2,080 & 2,670 & 3,340 & 3,900 & 4,760 & 5,060 & 3,770 & 3,670 & 3, & 4,300 & 4,290 & 230 \\
\hline & 1. 33 & 1,520 & 2,100 & 2,420 & 3,180 & 3,540 & 3,850 & 2,380 & 2,940 & 2,810 & 3,620 & 3,600 & 450 \\
\hline & b 1.73 & 1,330 & 1,600 & 1,940 & 2,470 & 4,340 & & 2,110 & 2,690 & & 30 & 3,560 & 200 \\
\hline \multirow[t]{2}{*}{12} & 1. 50 & 1,560 & 2,100 & 2,310 & 3,5 & 5,180 & 5,580 & 2,570 & 3,210 & 3,5 & & 5,450 & 4,480 \\
\hline & 1. 33 & 1,030 & 1,470 & 1,880 & 2,890 & 4,070 & 4,670 & 2,020 & 2,560 & 2,700 & 3, & 4,430 & 0,0 \\
\hline
\end{tabular}

INSULATED DURING FIRST 24 HOURS

\begin{tabular}{|c|c|c|c|c|c|c|c|c|c|c|c|c|c|}
\hline \multirow{4}{*}{1} & 1. 73 & 2,380 & 2,880 & 3,140 & 3,480 & 3,500 & 4,260 & 3,120 & 3,890 & 3,820 & 4,210 & 3,420 & 3,790 \\
\hline & 1. 50 & 1,800 & 2,100 & 2,430 & 2,660 & 2,720 & 3,200 & 2,550 & 3,890 & 2,930 & 3,630 & 2,420 & 3,320 \\
\hline & 1. 33 & 1,070 & 1,490 & 1,510 & 1,970 & 2,090 & 2,130 & 1,740 & 2,100 & 2,180 & 2,440 & 1,830 & 2,150 \\
\hline & 1. 73 & 1,690 & 2,600 & 3,160 & 3,470 & 4,430 & 5,380 & 3,080 & 3,620 & 3,710 & 4,160 & 4 , & 210 \\
\hline \multirow[t]{3}{*}{2} & 1. 50 & 1,260 & 2,070 & 2,120 & 2,890 & 3,140 & 4,020 & 2,080 & 2,510 & 2,420 & 3,170 & 3,520 & 380 \\
\hline & 1. 33 & 820 & 1,430 & 1,730 & 1,930 & 2,340 & 2,930 & 1,470 & 1,770 & 1,600 & 2,650 & 2,340 & 2,530 \\
\hline & 1. 73 & 1,270 & 1,990 & 2,430 & 3,020 & 4,140 & 4,830 & 2,670 & 3,060 & 2,670 & 3,590 & 3,860 & 4,210 \\
\hline \multirow[t]{3}{*}{3} & 1. 50 & 830 & 1,400 & 1,540 & 2,130 & 3,040 & 4,040 & 1,640 & 1,980 & 1,750 & 2,540 & 2,870 & 3,540 \\
\hline & 1. 33 & 480 & 860 & 1,140 & 1,610 & 2,160 & 2,720 & 1,030 & 1,650 & 1,190 & 1,810 & 2 , & 570 \\
\hline & 1. 73 & 2,150 & 3,010 & 3,280 & 3,700 & 4,270 & 5,340 & 3,180 & 3,850 & 3,730 & 4 , & 10 & 50 \\
\hline \multirow[t]{3}{*}{4} & 1. 50 & 1,180 & 2,080 & 2,590 & 3,230 & 3,560 & 4,440 & 2 , & 2,780 & 2 , & 3 , & 4 , & \\
\hline & 1. 33 & 870 & 1,610 & 1,860 & 80 & 2,760 & 3 , & 2,080 & 2,510 & 1,800 & 2,380 & 60 & 330 \\
\hline & 1. 73 & 1,600 & 2,790 & 2,910 & 3,590 & 4,240 & 4,660 & 3,080 & 3,690 & 3,620 & 4,300 & 90 & 510 \\
\hline \multirow[t]{3}{*}{5} & 1. 50 & 1,280 & 2,020 & 2,760 & 3,050 & 3,300 & 3,720 & 2,430 & 3,200 & 2,740 & 3,330 & 4,030 & 320 \\
\hline & 1. 33 & 830 & 1,670 & 2,200 & 2,480 & 2,690 & 3,040 & 1,880 & 2,550 & & 00 & 20 & \\
\hline & 1. 73 & 1,350 & 2,460 & 3,030 & 3,590 & 4,990 & 5,840 & 30 & 3,580 & 3,530 & 4 & 30 & 90 \\
\hline \multirow[t]{3}{*}{6} & 1. 50 & 1,000 & 1,950 & 2,420 & 3,400 & 3,890 & 4 , & 2, & 2,8 & 2 , & 30 & 60 & 30 \\
\hline & 1. 33 & 640 & 1 , & 1,910 & 2,600 & 3,000 & 3 , & 50 & 2,070 & 2,050 & 2,870 & 00 & 90 \\
\hline & 1. 73 & 2,040 & 3,100 & 3,850 & 4,160 & 5,180 & 5 , & 3,800 & 4,370 & 4,050 & 5,610 & & \\
\hline \multirow[t]{3}{*}{7} & 1. 50 & 1,470 & 2,300 & 3,150 & 4,000 & 4,080 & & & 3 , & 2,940 & 10 & & \\
\hline & 1. 33 & 1,020 & 1,730 & 1,600 & 2,070 & 2,120 & 10 & & 60 & 2,030 & 00 & & \\
\hline & 1. 73 & 1,870 & 2,670 & 3,450 & 3,840 & 4,820 & 5, & & 4,460 & 3,840 & 5 & 00 & 10 \\
\hline \multirow[t]{3}{*}{8} & 1. 50 & 1,270 & 2,050 & 3,340 & 3,820 & 3,720 & 4 , & 2 , & 3 , & 3 , & 4, & 50 & 00 \\
\hline & 1. 33 & 910 & 1 , & 2,290 & 3,1 & 3,160 & 3, & 1,950 & 2 , & 2 , & 3 , & 00 & 170 \\
\hline & 1. 73 & 2,120 & 3,350 & 3,640 & 4,210 & 4,290 & 5 , & 3,720 & 4,560 & 3,320 & 5,050 & 5,460 & 390 \\
\hline \multirow[t]{3}{*}{9} & 1. 50 & 1,030 & 2,070 & 3,130 & 3,800 & 4,480 & 5,240 & 2,240 & 3,240 & 2,960 & 3,780 & 20 & 20 \\
\hline & 1. 33 & 660 & 1,420 & 2,110 & 2,970 & 3,370 & 3,240 & 1,750 & 2,430 & 1,730 & 2 , & 90 & 50 \\
\hline & 1. 73 & 1,420 & 1,920 & 2,810 & 2,930 & 4,280 & 5 , & 2,240 & 3 , & 3,150 & 3,880 & 20 & 150 \\
\hline \multirow[t]{3}{*}{10} & 1. 50 & 940 & 1,560 & 1,870 & 2,530 & 3,460 & 4,240 & 1,770 & 2,640 & 2,240 & 3,010 & 3,370 & 30 \\
\hline & 1. 33 & 660 & 1,110 & 1,340 & 2,250 & 2,610 & 3 , & 70 & 1,750 & 1,580 & 2 , & 60 & 600 \\
\hline & 1. 73 & 1,930 & 2,850 & 3,590 & 4,010 & 4,590 & 5,800 & 3,610 & 3,950 & 3,670 & 4,860 & 4,770 & 5,110 \\
\hline \multirow[t]{3}{*}{11} & 1. 50 & 1,250 & 1,930 & 2,770 & 3,520 & 4,090 & 4,750 & 2,170 & 3,090 & 2,970 & 3,790 & & 30 \\
\hline & 1. 33 & 700 & 1,280 & 1,920 & 2,460 & 2,610 & 3,360 & 1,740 & 2,670 & 2,140 & 2,900 & & 20 \\
\hline & 1. 73 & 1,310 & 2,130 & 2,960 & 4,240 & 5,460 & 6,560 & 2,450 & 3,850 & 3,890 & 5,120 & & 40 \\
\hline \multirow[t]{2}{*}{12} & 1. 50 & 940 & 1,520 & 2,200 & 3,340 & 4,320 & 5,280 & 1,920 & 3,080 & 3,200 & 4,160 & 4,170 & \\
\hline & 1.33 & 580 & 1,140 & 1,650 & 2,910 & 3,430 & 3,870 & 1,410 & 1,740 & 1.930 & 3.480 & 3,350 & 0. \\
\hline
\end{tabular}

a Freezing in air at $5^{\circ}$ to $20^{\circ} \mathrm{F}, 16$ hours; thawing in water at about $70^{\circ} \mathrm{F}, 8$ hours.

b Cylinders for this cement, made at $110^{\circ} \mathrm{F}, C / W=1.73$, were honeycombed. 
The concretes in laboratory air storage also show a slight gain in strength between 1 year and 10 , but this gain is often just sufficient to compensate for the frequently noted slight decrease in strength between

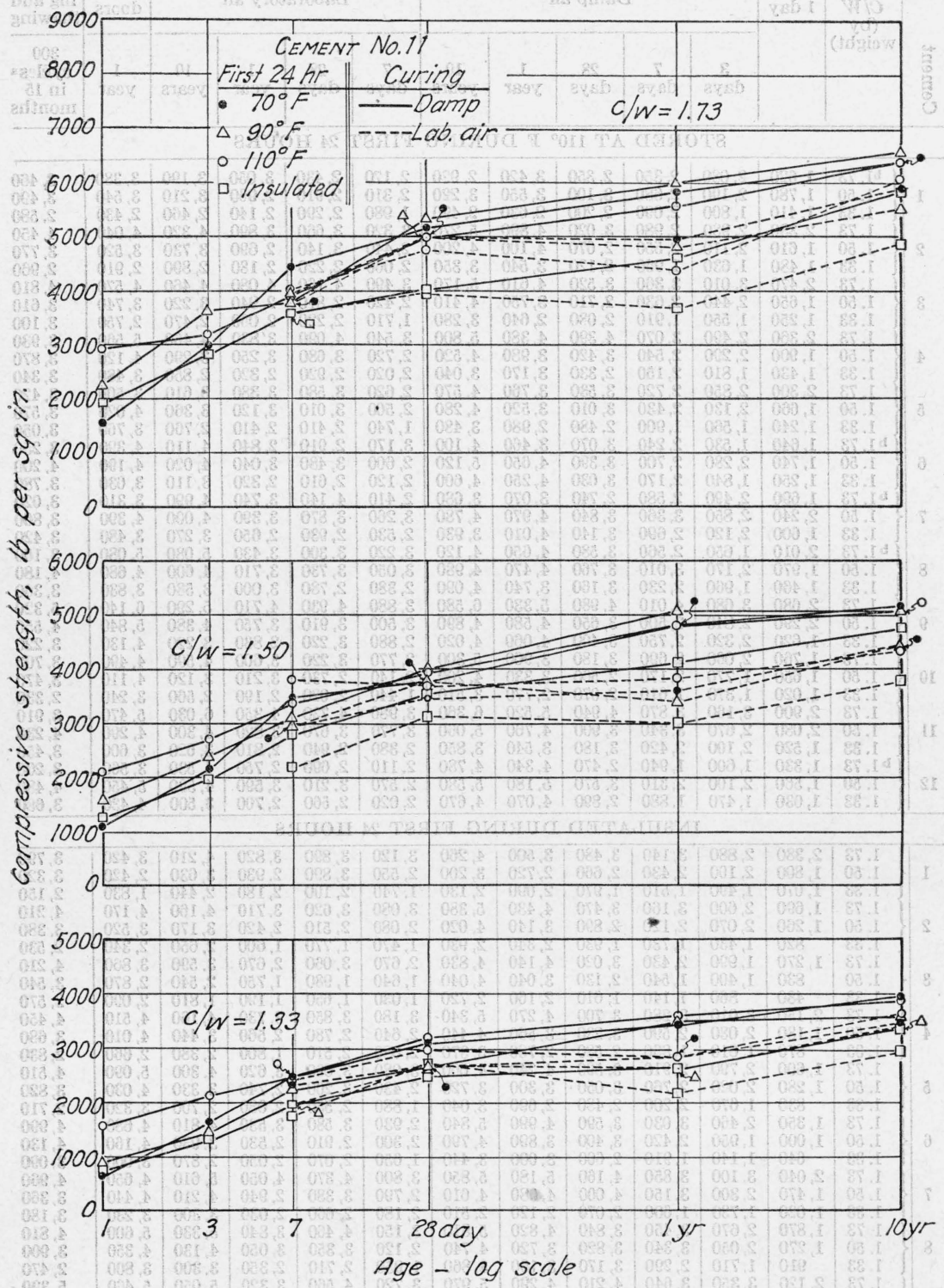

FIGURE 2.-Age-strength relations for cement 11 .

28 days and 1 year, so that generally the air-stored concretes are appreciably lower in strength at 10 years than those damp-cured.

It is of some interest to compare the strengths of the concretes of this investigation with those obtained by Withey ${ }^{2}$ for water-cured concretes

${ }_{-2}^{2}$ M. O. Withey, Some long-time tests of concrete, Proc. Am. Concrete Inst. 27, 547 (1931). 
made in 1910, and those of the PCA 1926 series cited by him. Such a comparison is made in table 3.

In order to make this comparison on an equal $C / W$ basis, it was necessary to interpolate the strength values for a $C / W$ of 1.60 between those for $C / W=1.73$ and $C / W=1.50$. The average strengths, for the twelve cements, for the $70^{\circ} \mathrm{F}$ initial, damp-cured concretes, were used. In addition, some as yet unpublished values recently obiained at this Bureau for various types of cement made in 1941 are included.

TABLE 3.-Compressive strengths of $1: 6$ concretes $\left(l b /\right.$ in. $\left.^{2}\right)$

\begin{tabular}{|c|c|c|c|c|c|}
\hline \multicolumn{4}{|c|}{ Age when tested } & \multirow{2}{*}{ Source of data } & \multirow{2}{*}{$\begin{array}{c}C / W \\
\text { (by } \\
\text { weight) }\end{array}$} \\
\hline 1 day & $1 \mathrm{mo}$ & $1 \mathrm{yr}$ & $10 \mathrm{yr}$ & & \\
\hline & \multirow{4}{*}{$\begin{array}{l}2,000 \\
3,000 \\
4,100 \\
4,600 \\
5,700 \\
4,250\end{array}$} & \multirow{4}{*}{$\begin{array}{l}3,200 \\
5,000 \\
5,000 \\
5,900 \\
5,700 \\
6,300\end{array}$} & 4,200 & \multirow{4}{*}{$\begin{array}{l}\text { Withey, series A, } 1910 \text { a } \\
\text { PCA, } 1926 \\
\text { NBS 1929-32, } 12 \text { high-early-strength cements } \\
\text { NBS 1941, } 5 \text { normal cements. } \\
\text { NBS 1941, } 3 \text { high-early-strength cements. } \\
\text { NBS 1941, } 4 \text { moderate-heat cements }\end{array}$} & \multirow{4}{*}{$\begin{array}{r}\text { 1. } 60 \\
\text { 1. } 60 \\
\text { 1. } 60 \\
\text { 1. } 66 \\
\text { 1. } 66 \\
\text { 1. } 66\end{array}$} \\
\hline 970 & & & 5,300 & & \\
\hline 1,060 & & & & & \\
\hline & & & (n) & & \\
\hline
\end{tabular}

a 6 - by 18 -in. cylinders.

b Interpolated from values for $C / W=1.73$ and 1.50 .

It is seen that the more recent cements (1941), even of the moderateheat type, attain strengths in 1 month about equal to those for the 1910 cement at 10 years. Similarly, the high-early-strength cements of 1941 gave strengths at 1 month exceeding those of the 1929-32 cements of this type at 10 years. The 1941 high-early-strength cements also showed appreciably higher 3 - and 7-day strengths than those for the 1929-32 cements (data not shown), though the 1-day strengths were not appreciably greater.

WASHINGTON, October 9, 1942. 